\title{
Integrating proteomics and transcriptomics for the identification of potential targets in early colorectal cancer
}

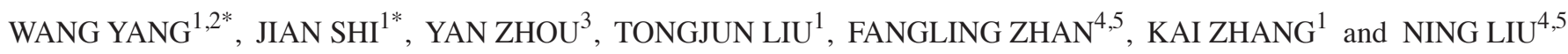 \\ ${ }^{1}$ Department of General Surgery, The Second Hospital of Jilin University, Changchun, Jilin 130041; \\ ${ }^{2}$ College of Clinical Medicine, Jilin University, Changchun, Jilin 130021; ${ }^{3}$ Department of Gastrointestinal Surgery, \\ The Second Hospital of Shandong University, Shandong $250000 ;{ }^{4}$ Central Laboratory, \\ The Second Hospital of Jilin University, Changchun, Jilin 130041; ${ }^{5}$ Key Laboratory of Zoonosis Research, \\ Ministry of Education, Jilin University, Changchun, Jilin 130062, P.R. China
}

Received March 16, 2019; Accepted June 20, 2019

DOI: $10.3892 /$ ijo.2019.4833

\begin{abstract}
Colorectal cancer (CRC) is one of the most common malignancies worldwide. At present, CRC can often be treated upon diagnosis at stage I or II, or when dysplasia is detected; however, $60-70 \%$ of cases are not diagnosed until they have developed into late stages of the disease or until the malignancy is identified. Diagnosis of CRC at an early stage remains a challenge due to the absence of early-stage-specific biomarkers. To identify potential targets of early stage CRC, label-free proteomics analysis was applied to paired tumor-benign tissue samples from patients with stage II CRC $(n=21)$. A total of
\end{abstract}

Correspondence to: Professor Ning Liu, Central Laboratory, The Second Hospital of Jilin University, 265 Ziqiang Street, Nanguan, Changchun, Jilin 130041, P.R. China

E-mail: liu_ning@jlu.edu.cn

Professor Kai Zhang, Department of General Surgery, The Second Hospital of Jilin University, 218 Ziqiang Street, Nanguan, Changchun, Jilin 130041, P.R. China

E-mail: zhangkai0628@126.com

${ }^{*}$ Contributed equally

Abbreviations: $\mathrm{BP}$, biological process; $\mathrm{CN}$, adjacent normal tissues; COAD, colon adenocarcinoma; CRC, colorectal cancer; CT, stage II CRC tissues; DEP, differentially expressed protein; ETFDH, electron-transfer flavoprotein-ubiquinone oxidoreductase; FC, fold change; FCGBP, Fc fragment of IgG binding protein; FDR, false discovery rate; GAR1, GAR1 ribonucleoprotein; GO, Gene Ontology; GS, gene significance; GSEA, Gene Set Enrichment Analysis; HPA, Human Protein Atlas; METTL7A, methyltransferase-like 7A; MM, molecular membership; NES, normalized enrichment score; PADI2, protein-arginine deiminase type-2; PPI, protein-protein interaction; PSAT1, phosphoserine aminotransferase 1; RNA-Seq, RNA-Sequencing; TCGA, The Cancer Genome Atlas; TGF- $\beta$, transforming growth factor- $\beta$; WGCNA, weighted correlation network analysis

Key words: CRC, WGCNA, label-free proteomics, transcriptome profiling, biomarkers
2,968 proteins were identified; corresponding RNA-Sequencing data were retrieved from The Cancer Genome Atlas-colon adenocarcinoma. Numerous bioinformatics methods, including differential expression analysis, weighted correlation network analysis, Gene Ontology and protein-protein interaction analyses, were applied to the proteomics and transcriptomics data. A total of 111 key proteins, which appeared as both differentially expressed proteins and mRNAs in the hub module, were identified as key candidates. Among these, three potential targets [protein-arginine deiminase type-2 (PADI2), $\mathrm{Fc}$ fragment of IgG binding protein (FCGBP) and phosphoserine aminotransferase 1] were identified from the pathological data. Furthermore, the survival analysis indicated that PADI2 and FCGBP were associated with the prognosis of CRC. The findings of the present study suggested potential targets for the identification of early stage CRC, and may improve understanding of the mechanism underlying the occurrence of CRC.

\section{Introduction}

In recent years, a large amount of epidemiological data has indicated that colorectal cancer (CRC) remains a common and lethal cancer (1-5); adenocarcinomas are a common type of CRC. At present, surgery remains the primary procedure for treating patients with CRC, as novel effective treatments have not yet been developed. Additionally, the recurrence rates following surgery in patients with CRC of stages I-III has been increasing (6). This may be overcome by the development of primary and secondary preventative strategies for $\operatorname{CRC}(7,8)$. As such, determining early CRC in high-risk populations and identifying targets to inhibit early CRC development are valid approaches. The occurrence and development of CRC are correlated with genetic mutations $(9,10)$. Identifying specific gene mutations and alterations in expression in tumor tissues have been the main focus of cancer research, and have led to developments in immunotherapy (11-13). Previous studies of CRC have mainly involved genomic and transcriptomics analyses (14-16). It is widely documented that proteins are key factors in biological processes; whether gene mutations can alter the expression of proteins is largely unknown. Therefore, 
a direct comparison of proteomic differences between tumor and normal tissues may provide insight into the development of CRC.

The Cancer Genome Atlas (TCGA) (17) contains extensive data regarding tumor genomes. In addition, the Human Protein Atlas (HPA) provides information regarding tissues, cells and pathology (18-20). TCGA and HPA are open-access, and have made considerable contributions to advances in tumor research. Weighted correlation network analysis (WGCNA) involves the separation of genes with the same expression pattern into the same module (21); thus, different modules represent sets of differentially expression pattern genes. Furthermore, these various modules could be associated with certain features of the data, and those linked to these characteristics of interest can be filtered out.

In the present study, stage II CRC was selected to represent early stage CRC. To identify potential targets of early CRC, TCGA-colon adenocarcinoma (COAD) stage II data were combined with the proteomic data of tumor and adjacent tissues from 21 patients with CRC for analysis.

\section{Materials and methods}

Clinical data of patients with stage II CRC. A total of 21 pairs (13 males and 8 females; age $\pm S D, 60.90 \pm 8.26$ years) of malignant tissues and adjacent benign or normal tissues from patients diagnosed with stage II CRC were obtained from The Tumor Tissue Bank at the Third Hospital of Jilin University between November 2015 and June 2016. All patients without hepatitis, tuberculosis and HIV did not receive radiotherapy or chemotherapy prior to surgery. Patients provided informed consent before surgery. The present study was approved by the Clinical Research Ethics Committee of the Second Hospital of Jilin University. The experimental scheme applied in the present study is presented in Fig. 1.

Protein extraction and filter-aided tryptic digestion. Frozen tissues ( $100 \mathrm{mg}$ each) were placed into 2-ml screw-cap tubes pre-filled with ceramic beads (1.4 mm; Roche Diagnostics), followed by the addition of $1 \mathrm{ml}$ lysis buffer [ $8 \mathrm{M}$ urea, $100 \mathrm{mM}$ Tris, $50 \mathrm{mM}$ dithiothreitol (DTT), $1 \mathrm{mM}$ phenylmethylsulphonyl fluoride and protease inhibitor cocktail]. Tissue sample homogenization was then performed on a MagNA Lyser Instrument (Roche Diagnostics). The homogenous lysates were centrifuged at $40,000 \times \mathrm{g}$ at $4^{\circ} \mathrm{C}$ for $1 \mathrm{~h}$ to isolate the proteins. The protein content of each sample was determined and adjusted by adding the lysis buffer. Subsequently, stage II CRC tissues (CT) and paired adjacent normal tissues $(\mathrm{CN})$ were pooled. The pooled samples were aliquoted and stored at $-80^{\circ} \mathrm{C}$ until use; the two pooled protein samples $(\mathrm{CT}$ and $\mathrm{CN})$ were then subjected to proteomics analyses. The protein samples were thawed on ice and further processed using a filter-aided tryptic digestion method, as previously described (22). Briefly, samples were concentrated by centrifugation at $15,000 \mathrm{x} \mathrm{g}$ at room temperature for $20 \mathrm{~min}$ in $1.5 \mathrm{ml} 10 \mathrm{kDa}$ ultrafiltration centrifuge tubes (Pall Corporation). Then, the concentrated samples $(<20 \mu \mathrm{l})$ were diluted with $200 \mu \mathrm{l}$ buffer $(8 \mathrm{M}$ urea and $50 \mathrm{mM}$ Tris) supplemented with $10 \mathrm{mMDTT}$ and stored at $55^{\circ} \mathrm{C}$ for $1 \mathrm{~h}$. The samples were centrifuged at $15,000 \mathrm{xg}$ at room temperature for $20 \mathrm{~min}$ and then mixed with $200 \mu \mathrm{l}$ buffer $(8 \mathrm{M}$ urea and $50 \mathrm{mM}$ Tris) supplemented with $20 \mathrm{mM}$ iodoacet- amide. Following alkylation in the dark at $37^{\circ} \mathrm{C}$ for $30 \mathrm{~min}$, the protein samples underwent buffer-exchange twice with $50 \mathrm{mM}$ ammonium bicarbonate solution. Digestion of the protein samples was initiated by adding L-1-Tosylamide-2-phenylethyl chloromethyl ketone-modified sequencing-grade trypsin (Promega Corporation) at an enzyme/protein ratio of 1:100. The reaction was conducted at $37^{\circ} \mathrm{C}$ for $\geq 15 \mathrm{~h}$ and quenched by adding $10 \%$ formic acid to a final concentration of $1 \%$. The tryptic digests were subject to centrifugation at $15,000 \mathrm{x} \mathrm{g}$ at room temperature for $20 \mathrm{~min}$, and the filtrates were collected and de-salted with C18 Ziptips (EMD Millipore). The purified tryptic peptides were freeze-dried and stored at $-80^{\circ} \mathrm{C}$ until use.

Nano liquid chromatography (LC)-tandem mass spectrometry (MS/MS). The tryptic peptide samples, solubilized in $20 \mu \mathrm{l} 0.1 \%$ (vol/vol) trifluoroacetic acid, were analyzed using a TripleTOF5600+ mass spectrometer (AB Sciex) coupled with an Eksigent nanoLC system (AB Sciex). The peptide mixture was separated using a C18 capillary column (ChromXP; SCIEX; $150 \mathrm{~mm}$ x $75 \mu \mathrm{m} \mathrm{x}$ $3.0 \mu \mathrm{m}$ ) at $300 \mathrm{nl} / \mathrm{min}$, using a $120 \mathrm{~min}$ gradient rendered by solvents A (2\% acetonitrile $/ 0.1 \%$ formic acid) and B (98\% acetonitrile/0.1\% formic acid). The entire gradient comprised 1-35\% solvent B for $90 \mathrm{~min}, 35-80 \%$ solvent B for $15 \mathrm{~min}$, and $85 \%$ solvent B for $15 \mathrm{~min}$. The mass spectrometer, fitted with a PicoView Nanospray source, which does not require a nebulizer gas (PV400; New Objective), was operated under the positive ion mode. Complete MS spectra were acquired for the mass range of $350-1,250 \mathrm{~m} / \mathrm{z}$. The proteomics data were collected using information-dependent-acquisition mode by selecting 10 most abundant ions for MS/MS fragmentation under the following conditions: The number of charged ions is 2-5 and the collision energy is applied in the mode of Rolling Collision Energy.

Database searches and data processing. The collected data files (.wiff) were transferred to a data processing workstation. MS data analysis software ProteinPilot 5.0 (AB Sciex) was used for protein database searching against the SwissProt database (ftp://ftp.uniprot.org/pub/databases/uniprot/current release/knowledgebase/complete/uniprot_sprot.fasta.gz). The parameters were set as follows: i) The protease was selected as trypsin; ii) alkylation of Cys by iodoacetamide; and iii) biological modifications were selected as the ID Focus. The resulting group files were converted into mzIDentML format using the GroupFileExtractor tool affiliated with ProteinPilot (https://download.sciex.com/ProteinPilot_502-relNotes.pdf). Scaffold (version Scaffold_4.8.4; Proteome Software) was used to validate MS/MS-based peptide and protein identifications. Peptide identifications were accepted if they could be established at $>73.0 \%$ probability to achieve a false discovery rate (FDR) $<1.0 \%$ by the Scaffold Local FDR algorithm. Protein identifications were accepted if they could be established at $>5.0 \%$ probability to achieve an FDR $<1.0 \%$ and contained $\geq 2$ identified peptides. Proteins that contained similar peptides and could not be differentiated based on MS/MS analysis alone were grouped to satisfy the principles of parsimony. Proteins sharing significant peptide data were grouped into clusters. Spectral counting, which refers to the total number of spectra identified for a protein, was used to quantitatively estimate protein abundance in the proteomics dataset. 


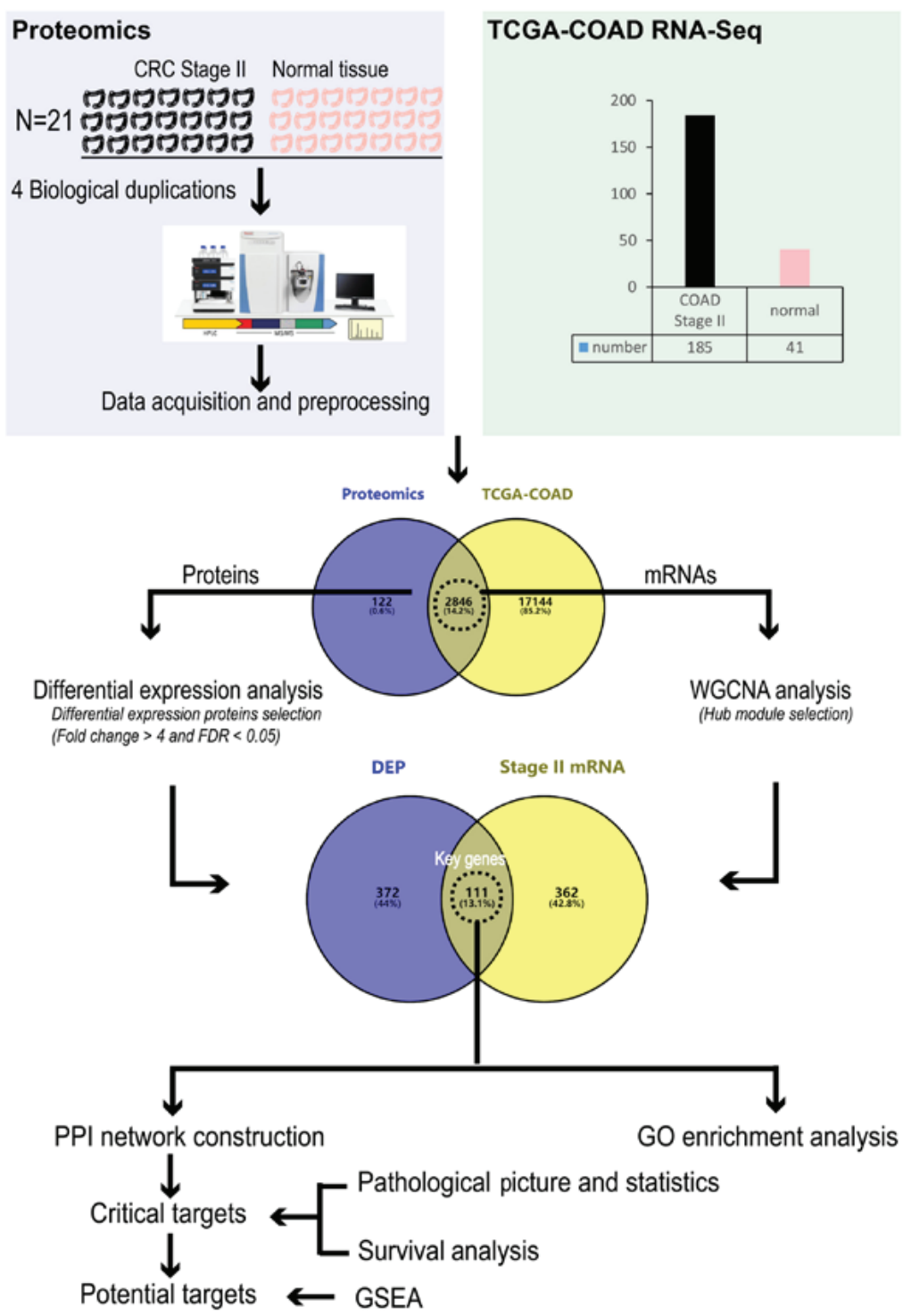

Figure 1. Flow chart for identifying potential targets of stage II CRC. CRC, colorectal cancer; TCGA, The Cancer Genome Atlas; COAD, colon adenocarcinoma; RNA-Seq, RNA-Sequencing; FDR, false discovery rate; WGCNA, weighted correlation network analysis; DEP, differentially expressed protein; PPI, protein-protein interaction; GO, Gene Oncology; GSEA, Gene Set Enrichment Analysis.

RNA sequencing data acquisition. The mRNA expression profiles of TCGA-COAD- Fragments Per Kilobase of transcript per Million mapped reads (FPKM) were downloaded from TCGA (https://cancergenome.nih. gov/) (17). The datasets numbers are presented in Fig. S1. In the present study, there were 226 samples, including 41 normal tissue normal samples and 185 COAD stage II samples. The Encyclopedia of DNA Elements (23) (GRCh38; V22) catalog (https://www.gencodegenes.org/releases/22.html) was used as a reference to identify mRNAs. Briefly, 19,990 mRNAs from the RNA-Sequencing (RNA-Seq) data were extracted. The data were downloaded from R Studio v1.2.1335 (https://www. rstudio.com/products/rstudio/download/\#download) using R package TCGAbiolinks (24).

Differentially expressed protein (DEP) selection. The 'edgeR' package $(25,26)$ was used to select DEPs between $\mathrm{CT}$ and $\mathrm{CN}$. The FDR P-value was corrected by the Benjamini-Hochberg method (26). The selection criteria of DEPs were FDR P-value $<0.05$ and fold change (FC) $>4$.
Then, the distribution of DEPs were presented as heatmaps and volcano plots using the ggplot2 package (27) in R.

Construction of a scale-free network construction by WGCNA. To link proteomics data with mRNA data, only genes overlapping in the proteomics and mRNA data were selected for subsequent WGCNA analysis. WGCNA (21) was used to analyze RNA-Seq data and two main parts comprised this analysis. In the first part, the correlation coefficients between any two mRNAs were calculated to determine whether two mRNAs have similar expression patterns. WGCNA employs correlation coefficient weights to construct a scale-free network. The weighted gene co-expression network stresses high correlations at the cost of low correlations by increasing the absolute value of the correlation to a power $\beta \geq 1$ (soft thresholding). The best-suited soft threshold value $(\beta)$ was selected to build the scale-free network. Then, the scale-free network distribution could be tested. In the second part, the hierarchical clustering tree was constructed by using the correlation coefficients between mRNAs. Based on the 
weighted correlation coefficient of mRNAs, mRNAs were classified according to their expression pattern, and mRNAs with similar patterns were grouped into the same module. Subsequently, the module-trait association was determined by combining the sample information. A correlation test P-value or a regression-based P-value was used for evaluating the statistical significance between $\mathrm{x}_{\mathrm{i}}$ and trait (21).

Gene Ontology (GO) enrichment analysis of key genes. DEPs from proteomics data were screened; in addition, the hub gene module of mRNAs was determined. Genes that appeared as both DEPs and mRNAs of the hub module were defined as key genes. GO enrichment analysis (28) comprised biological process (BP), cellular component and molecular function; BP was selected to understand the general function of key genes. Then, 'clusterProfiler' (29) was used for GO enrichment analysis. The top 15 GO terms with the highest significance following enrichment were chosen.

Construction of a protein-protein interaction (PPI) network. A PPI network of key genes was constructed using the STRING v11.0 $(30,31)$. Subsequently, Cytoscape v3.6.0 (https://cytoscape.org/download.html) was used to present the PPI network; default settings of the Cytoscape MCODE plug-in (http://apps.cytoscape.org/apps/mcode) were applied. The key cluster and seed node of each cluster were then obtained. These seed nodes were considered as critical targets in the PPI network.

Pathological images and survival analysis. The HPA (https://www.proteinatlas.org/) is a free and open database comprising three sub-atlases: The tissue, cell and the pathology atlases, which provide a large amount of public data available for cancer research (18-20). To screen clinically important molecules from critical targets, pathological images were obtained and survival analysis was conducted based on TCGA-COAD clinical data in the HPA database (https://www.proteinatlas. org/humanproteome/pathology/colorectal+cancer). Normal colonic tissue contains epithelial cells, glandular cells and peripheral nerves/ganglia. Of note, the tumor employed for analysis was adenocarcinoma; thus, the clinical significance of each potential target was determined based on the immunohistochemistry analysis of glandular cells in normal tissue. Additionally, Kaplan-Meier analysis revealed a correlation between the expression levels of mRNA and patient survival; P-values were obtained using a log-rank test. Based on the FPKM value of each gene, the patients were classified into two groups and their prognoses were examined. In the analysis, genes with low expression were excluded, such as those with a median expression among samples with FPKM $<1$. The prognosis of each group of patients was examined by Kaplan-Meier survival estimators, and the survival outcomes of the two groups were compared by log-rank tests. To choose the best FPKM cut-offs for grouping the patients most significantly, all FPKM values from the 20th to 80th percentiles were used to group the patients; significant differences in the survival outcomes of the groups were examined and the value yielding the lowest log-rank P-value was selected (https://www. proteinatlas.org). The data used for the survival analysis included all the TCGA-COAD data in the HPA database.

Statistical analysis. Using the 'ggstatsplot' package (https://github.com/IndrajeetPatil/ggstatsplot/issues) in R, further statistical analysis of the critical targets was conducted to interpret pathological images and survival results. P-values were obtained by an independent samples t-test. The statistical significance cut-off level was $\mathrm{P}<0.05$.

Gene Set Enrichment Analysis (GSEA) and protein classes of potential targets. To verify the biological function of potential targets, their expression levels were used to classify samples into high- and low-expression groups. Kyoto Encyclopedia of Genes and Genomes (v6.2) (32) enrichment analysis was conducted using GSEA v3.0 $(33,34)$. The mRNA data of TCGA-COAD stage II were used for the GSEA, and these mRNAs were present in both the proteomics and transcriptomics data. The parameters of the software were set to default. The normalized enrichment score (NES) value and the P-value of the enriched pathways were used to confirm the accuracy of the results. INESI $>1$ and $\mathrm{P}<0.05$ were used to filter the pathways. Then, the 'ggplot2' and 'ggrepel' packages (https://github.com/slowkow/ggrepel/issues) were applied to present the results in the $\mathrm{R}$ platform. Meanwhile, the protein classes were queried using the HPA database (https://www. proteinatlas.org/).

\section{Results}

Distribution of protein expression regulation. In total, 2,968 proteins were identified in the stage II CRC proteomics data. Most of the identified proteins (2,846 items) were identified in TCGA-COAD transcriptome data (Fig. 1). 328 upregulated DEPs and 155 downregulated DEPs $(\mathrm{P}<0.05 ; \mathrm{FC}>4)$ were reported (Fig. 2).

Construction of a scale-free network and screening for hub modules. In the present study, 2,846 coincident genes coexisted in stage II CRC proteomics and TCGA-COAD data. The hierarchical clustering tree revealed the similarity of different samples. The results demonstrated that the cancer tissue and normal sample had apparent heterogeneity (Fig. S1). In addition, WGCNA was used to construct a scale-free network, which was built with $\beta=8$ (Fig. S2A-D). A total of four gene modules (blue, brown, turquoise and grey) were generated; the majority of the genes were summarized into the 'turquoise module' (Fig. S2E).

According to the module-trait association, the 'blue' module with the highest correlation coefficient was selected for subsequent analysis (Fig. 3A). Gene significance (GS) was used to measure the degree of association between protein and trait. Module membership (MM) was used to determine the location of a global network. GS versus MM reflected the relationship between characteristics and proteins. The results revealed that the 'blue module' was essential (Fig. 3B and C).

$\mathrm{GO}$ enrichment analysis of key genes. There were 111 key genes derived from the overlapping of DEPs and mRNAs of the 'blue' module. Then, BPs associated with these genes were identified by GO enrichment analysis; only the top 15 most significant GO terms were used to determine the general function of these genes. A total of $12 / 15 \mathrm{GO}$ terms were mainly involved in immunity, including 'neutrophil degranulation', 'neutrophil activation involved in immune response', 'neutrophil activation', 'neutrophil mediated immunity', 'lymphocyte mediated immunity', 'leukocyte migration', 'B cell mediated immunity', 'immunoglobulin mediated immune response', 'protein activation cascade', 'complement activation, classical 
A

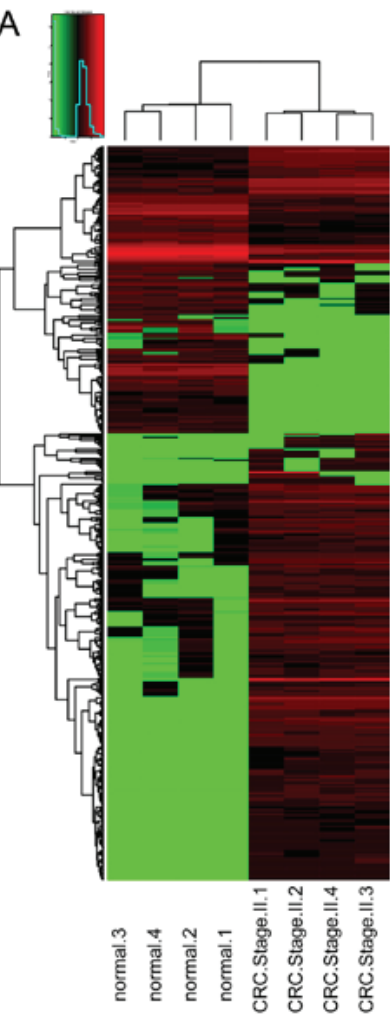

$\mathrm{B}$

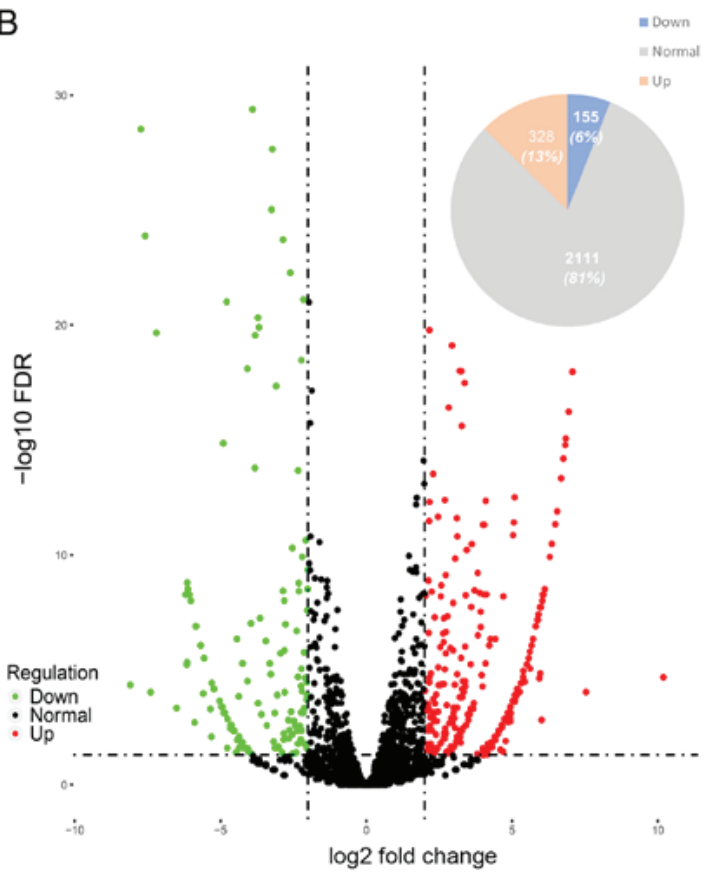

Figure 2. Distribution of DEPs. (A) A heatmap of DEPs of stage II CRC. The horizontal axis represents the name of the sample, while the vertical axis indicates the genes. (B) A volcano plot of DEPs of stage II CRC. The horizontal axis represents the 'log2Fold Change', while the vertical axis indicates the '-log10FDR'. $\mathrm{P}<0.05$; Fold Change $>4$. DEPs, differentially expressed proteins; CRC, colorectal cancer; FDR, false discovery rate.

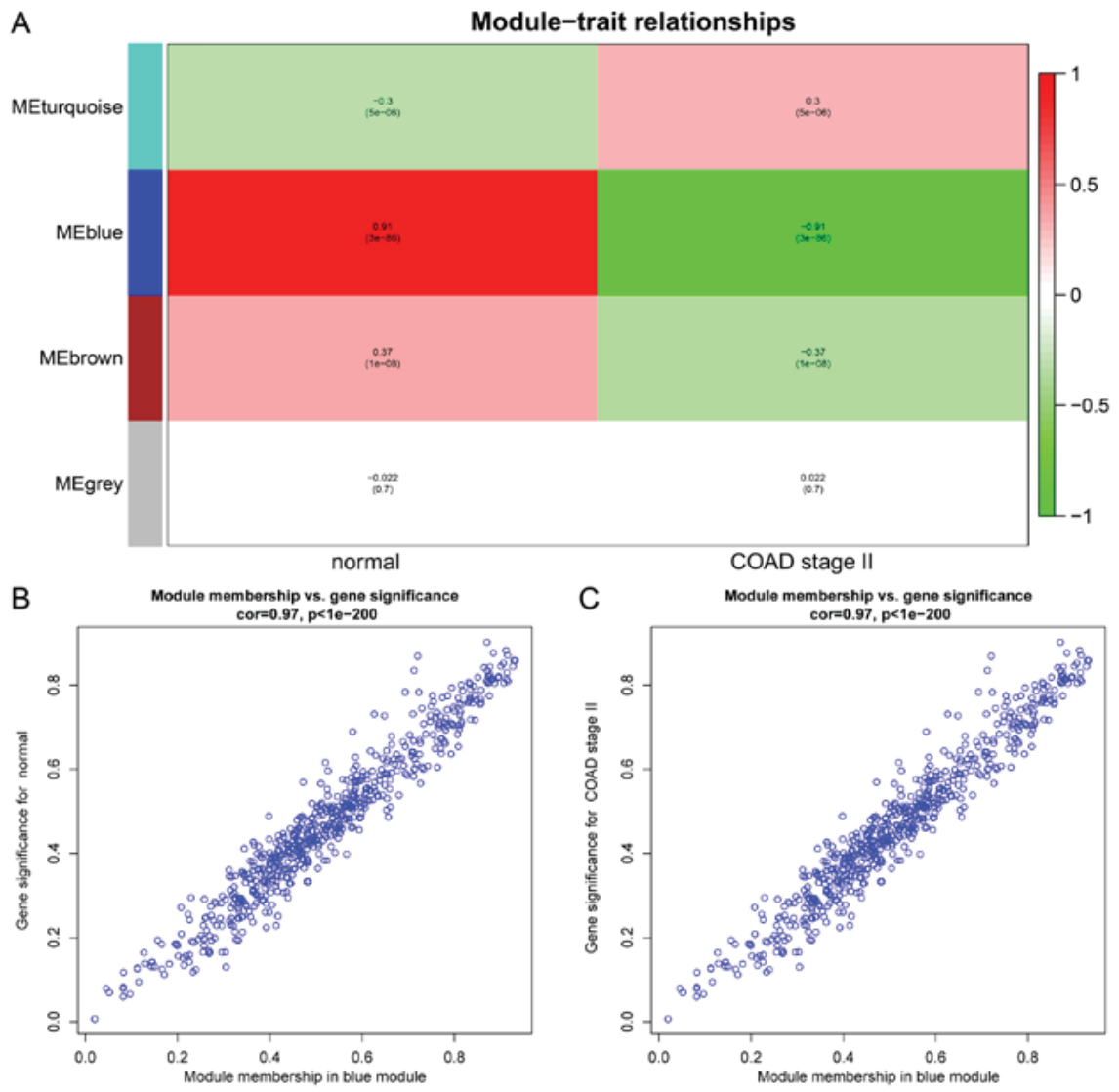

Figure 3. Module-trait association and members of a module versus gene significance of the hub module. (A) Module-trait association of data. The first row of each cube represents the correlation coefficient between the module and the trait, and the second row indicates the significance of the correlation coefficient. The correlation coefficient is presented by the color of the cube; red indicates positive correlation, while green represents negative correlation. (B) Members of the blue modules vs. gene significance for normal. (C) Members of the blue module vs. gene significance for stage II COAD. COAD, colon adenocarcinoma; ME, module eigengene. 


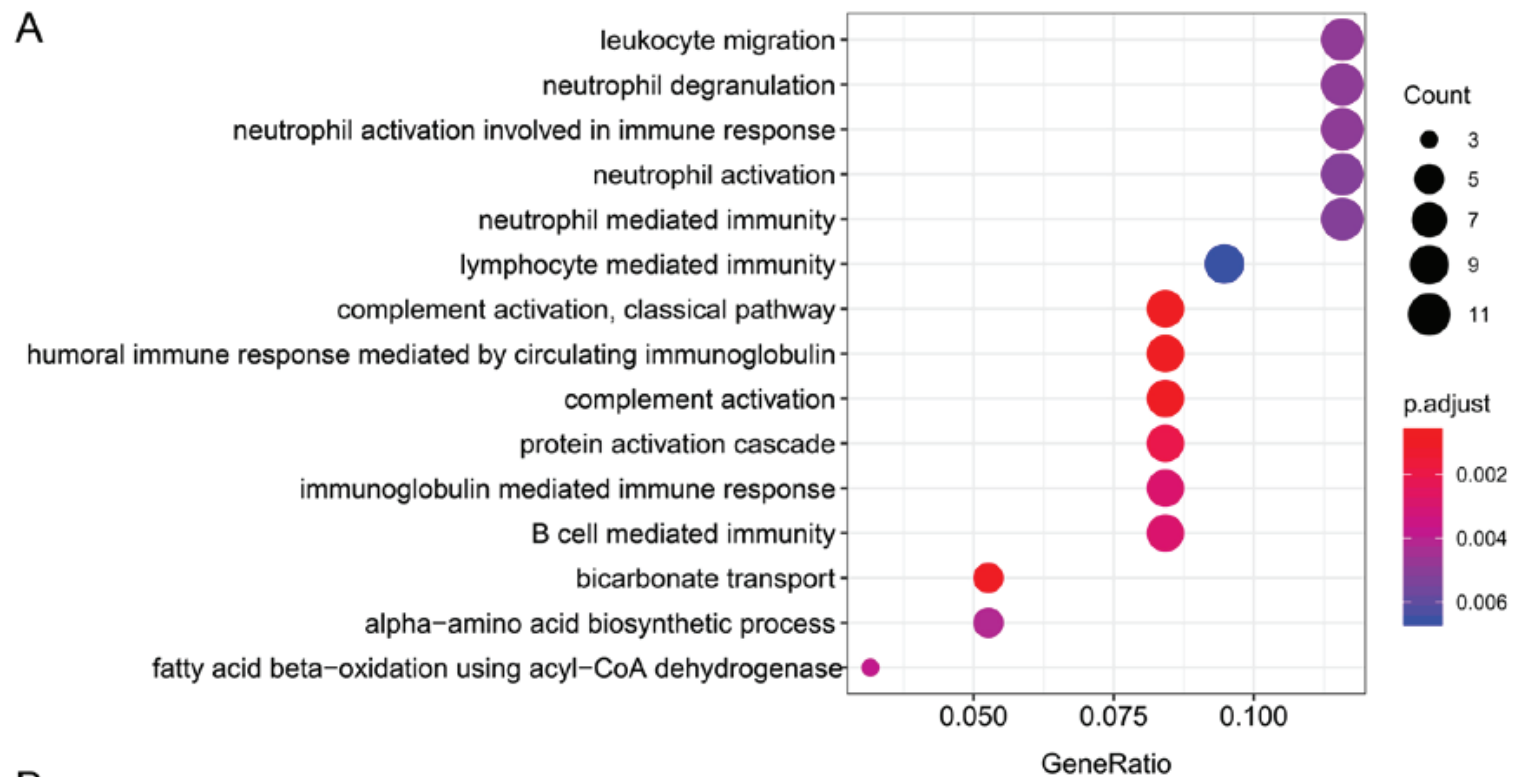

B
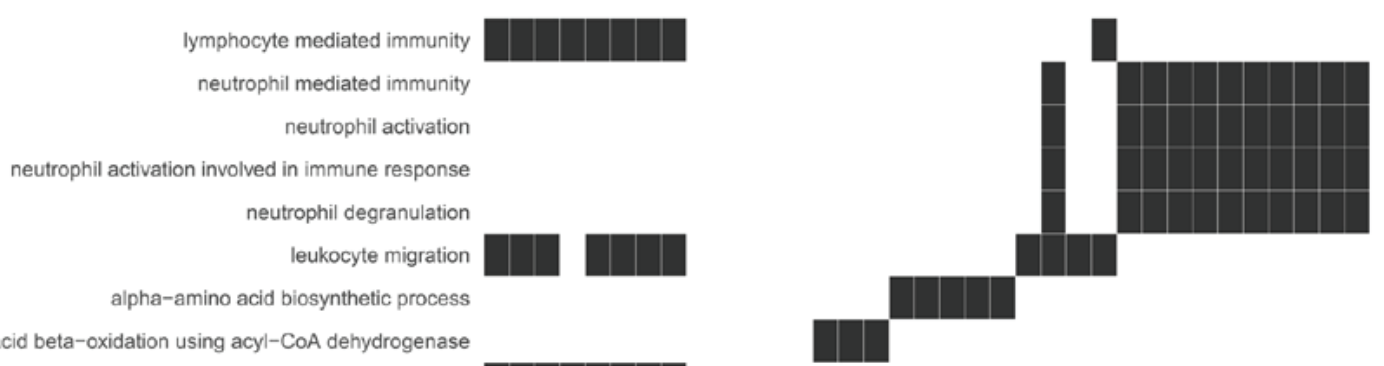

immunoglobulin mediated immune response

protein activation cascade

complement activation

bicarbonate transport

humoral immune response mediated by circulating immunoglobulin

complement activation, classical pathway
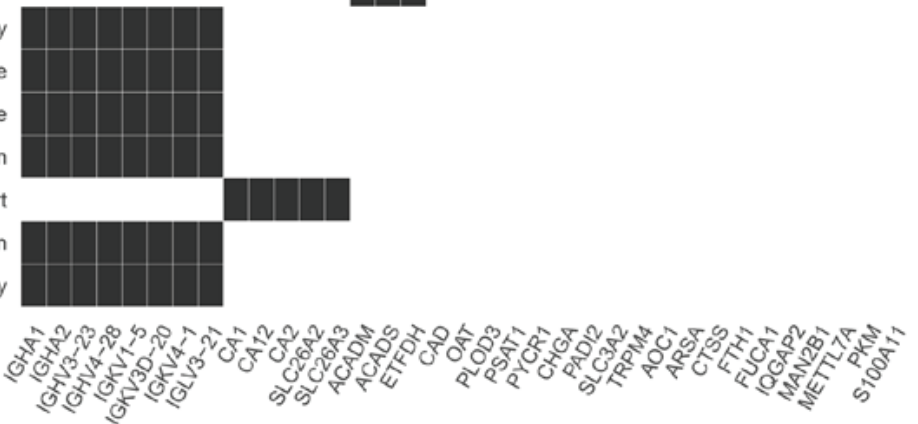

Figure 4. GO enrichment analysis of key genes. (A) Top 15 GO terms listed by the count of genes associated with the GO term. The color of the dot indicated the adjusted P-value; red indicated higher significance in association with the GO term. (B) Top $15 \mathrm{GO}$ terms. The horizontal axis presents the genes associated with the GO term; genes of the same GO term were clustered. GO, Gene Ontology.

pathway', 'humoral immune response mediated by circulating immunoglobulin' and 'complement activation'. 'Complement activation' was the most significant. The remaining 3/15 GO terms were involved in metabolic pathways, including 'bicarbonate transport' with the highest significance (Fig. 4).

Construction of PPI networks and identification of critical targets. Based on the STRING database, a PPI network of associated key genes was built. Using the MCODE plug-in, six clusters were selected in the PPI network, including protein-arginine deiminase type-2(PADI2), Fc fragment of IgG binding protein (FCGBP), GAR1 ribonucleoprotein (GAR1), phosphoserine aminotransferase 1 (PSAT1), electron-transfer flavoprotein-ubiquinone oxidoreductase (ETFDH) and methyltransferase-like 7A (METTL7A), which were the seed nodes in each cluster (Fig. 5).

Pathological images and statistics. To verify the clinical role of these critical targets, pathological images (Fig. 6) and statistical analysis (Fig. 7) of PADI2 ( $<<0.001$ in RNA-Seq and $\mathrm{P}<0.001$ in proteomics), FCGBP $(\mathrm{P}<0.001$ in RNA-Seq and $\mathrm{P}<0.001$ in proteomics), GAR1 ( $\mathrm{P}<0.001$ in RNA-Seq and $\mathrm{P}=0.020$ in proteomics), $\mathrm{PSAT1}(\mathrm{P}<0.001$ in RNA-Seq and $\mathrm{P}=0.003$ in proteomics) and ETFDH ( $\mathrm{P}<0.001$ in RNA-Seq and $\mathrm{P}=0.108$ in proteomics) were obtained, but not for METTL7A. Of note, the pathological images of PADI2, FCGBP and PSAT1 were notably different from those of normal colon tissues (Figs. 6 and 7). Additionally, PADI2 ( $\mathrm{P}=0.0084)$ and FCGBP $(\mathrm{P}=0.0031)$ were associated with the prognosis of COAD (Fig. 8).

Biological function and protein classes of potential gene targets. The results of the GSEA indicated that alterations in PADI2 were most likely to affect 'fatty acid metabolism', changes in FCGBP were most likely to influence 'ubiquitin mediated proteolysis' and alterations in PSAT1 were most likely to lead to changes in the 'purine metabolism' pathway (Fig. 9). In the HPA database, PADI2 was predicted as an intracellular 


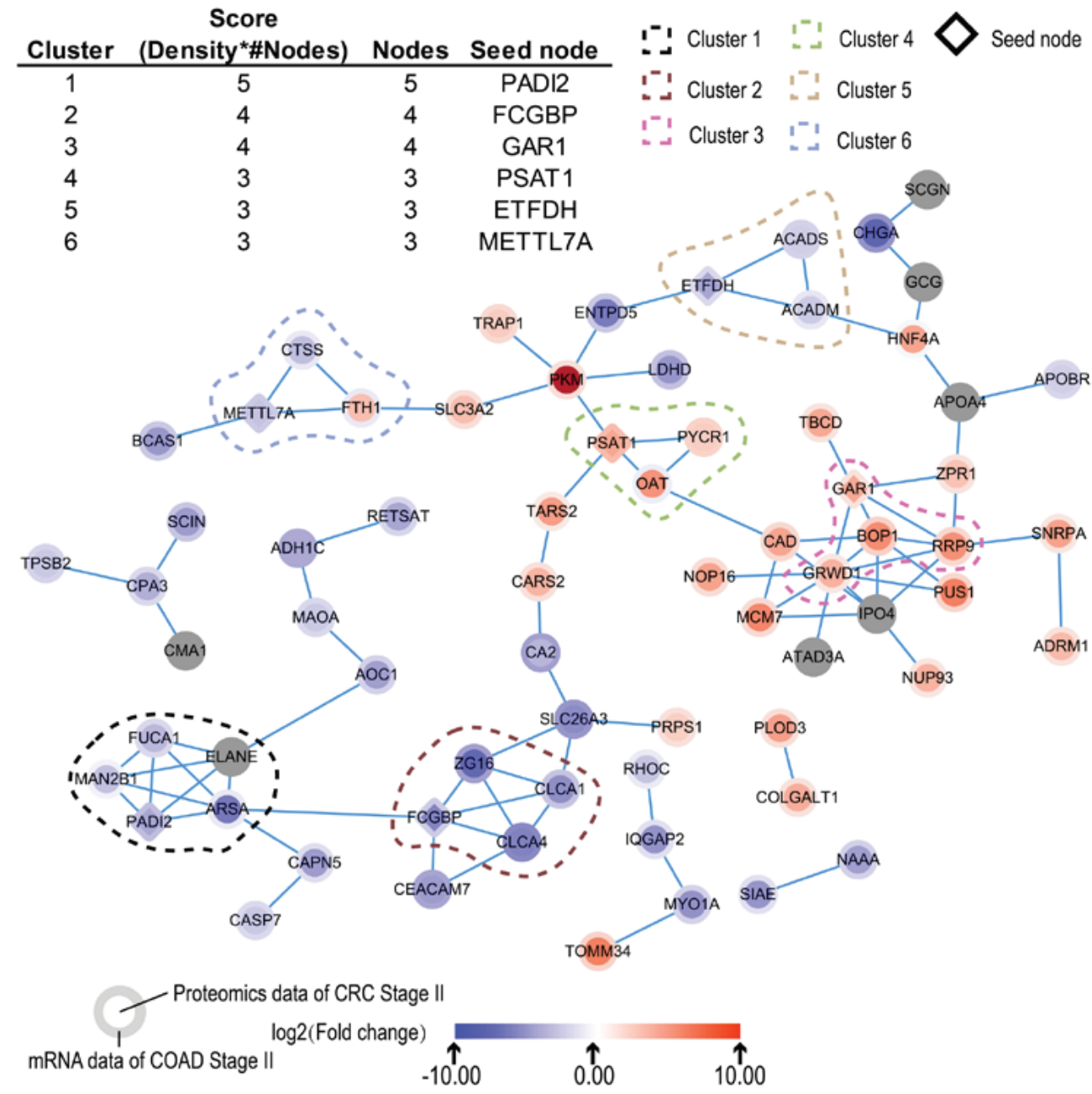

Figure 5. Protein-protein interaction network of stage II CRC. The six key clusters are presented as different colors; the seed node of the cluster is presented as a diamond. The color of the inner ring of the node indicates the fold change in proteomics data. The intensity of the outer circle indicates the fold change in the mRNA. CRC, colorectal cancer; COAD, colon adenocarcinoma; PADI2, protein-arginine deaminase type-2; FCGBP, Fc fragment of IgG binding protein; GAR1, GAR1 ribonucleoprotein; PSAT1, phosphoserine aminotransferase; ETFDH, electron-transfer flavoprotein-ubiquinone oxidoreductase; METTL7A, methyltransferase-like 7A.

protein; FCGBP was predicted as a secreted protein and an intracellular protein, while PSAT1 was determined to be a plasma protein and a predicted intracellular protein.

\section{Discussion}

In the present study, potential targets (PADI2, FCGBP and PSAT1) were identified in stage II CRC. In addition, pathological images and survival analysis confirmed the function of these targets. A large amount of RNA-Seq data were accumulated from previous studies (35-37); at present, few CRC-related proteomics analyses have been conducted to the best of our knowledge. In the present study, WGCNA was conducted to analyze RNA-Seq data. WGCNA focused on the expression pattern, while the differential expression analysis revealed differences between tumor and normal tissue. The information obtained from the data was extracted by selecting the most appropriate method of analysis. Although the number of samples was low, differences in the proteomics data of 21 patients with stage II CRC were analyzed. In addition, stage II CRC stage-related key genes were screened by determining overlapping DEPs and mRNAs in the hub module. Of note, proteins mainly execute biological processes; alterations in proteins can reflect dysfunctional biological processes in cancer. Proteomics and RNA-Seq data were combined prior to screening potential targets in stage II CRC. The GO enrichment analysis revealed that the activation of the immune system is vital in stage II CRC, involving regulation of neutrophil and 'complement activation' in particular. On the contrary, inflammatory cells are an essential part of the tumor microenvironment, especially neutrophils $(38,39)$. A previous study demonstrated that the density of tumor-associated neutrophils in late CRC was significantly lower than early stage CRC (40). Of note, it has been suggested that complement activation may facilitate the development of CRC (41-43). In addition, the presence of neutrophils was associated with the prognosis of this disease (44-46).

In the present study, the PPI network revealed the expression of components between the proteomics and RNA-Seq data to be similar. A total of six critical targets (PADI2 in cluster 1 and FCGBP in cluster 2) were selected from the PPI network that was constructed with key genes; five of the key targets were identified in the HPA database. Furthermore, PADI2, FCGBP and PSAT1 notably differed between the pathological 
A

\begin{tabular}{ll}
\multicolumn{3}{c}{ Normal colon } \\
\hline Patient ID & 4510 \\
Sex & Male \\
Age & 47 \\
Stain & High \\
Intensity & Strong \\
Quantity & $>75 \%$ \\
& \\
\hline & CRC \\
\hline Patient ID & 4751 \\
Sex & Male \\
Age & 57 \\
Stain & Not detected \\
Intensity & Weak \\
Quantity & $<25 \%$
\end{tabular}

C

\begin{tabular}{ll|}
\multicolumn{3}{c}{ Normal colon } \\
\hline Patient ID & 4510 \\
Sex & Male \\
Age & 47 \\
Stain & High \\
Intensity & Strong \\
Quantity & $>75 \%$ \\
& \\
\hline & CRC \\
\hline Patient ID & 4735 \\
Sex & Male \\
Age & 62 \\
Stain & High \\
Intensity & Strong \\
Quantity & $>75 \%$
\end{tabular}

E

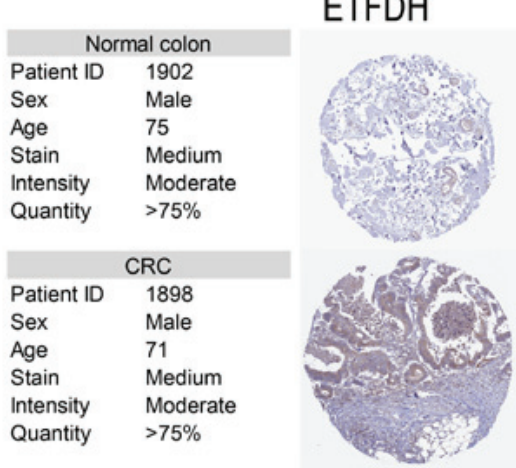

B

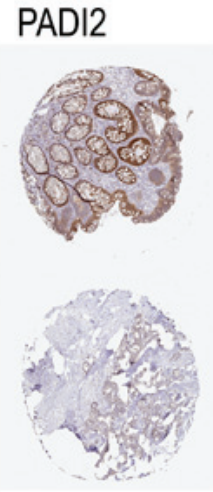

GAR1

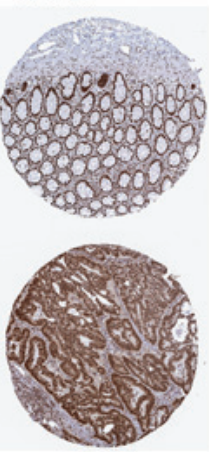

ETFDH

\begin{tabular}{ll}
\multicolumn{2}{c}{ Normal colon } \\
\hline Patient ID & 1832 \\
Sex & Female \\
Age & 61 \\
Stain & High \\
Intensity & Strong \\
Quantity & $>75 \%$ \\
& \\
\hline & CRC \\
\hline Patient ID & 1958 \\
Sex & Female \\
Age & 84 \\
Stain & Not detected \\
Intensity & Negative \\
Quantity & Negative
\end{tabular}

FCGBP

D

\begin{tabular}{ll}
\multicolumn{2}{c}{ Normal colon } \\
Patient ID & 2944 \\
Sex & Female \\
Age & 80 \\
Stain & Not detected \\
Intensity & Weak \\
Quantity & $<25 \%$ \\
& \\
& CRC
\end{tabular}

Patient ID $\quad 2106$

Sex Female

Age $\quad 86$

Stain High

Intensity Strong

Quantity $\quad>75 \%$

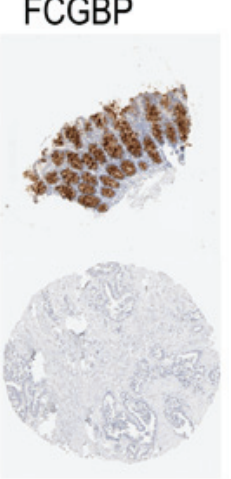

PSAT1

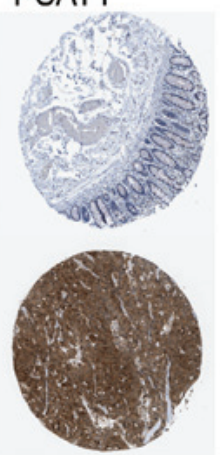

Figure 6. Pathological images of critical targets. The pathological and normal images of (A) PADI2, (B) FCGBP, (C) GAR1, (D) PSAT1 and (E) ETFDH. The information was obtained from the Human Protein Atlas. Magnification, x20. PADI2, protein-arginine deaminase type-2; FCGBP, Fc fragment of IgG binding protein; GAR1, GAR1 ribonucleoprotein; PSAT1, phosphoserine aminotransferase; ETFDH, electron-transfer flavoprotein-ubiquinone oxidoreductase; CRC, colorectal cancer.

images and the normal tissue images; PADI2 and FCGBP were associated with prognosis. Through signal-gene GSEA, PADI2 tended to affect 'fatty acid metabolism'; FCGBP changes were most likely to influence 'ubiquitin mediated proteolysis'; and PSAT1 changes were most likely to lead to changes in the 'purine metabolism' pathway.

Certain findings of the present study were consistent with previous literature. PADI2 was downregulated in CRC tissue; the occurrence of early CRC has been associated with downregulated PADI2 $(47,48)$ and indicated poor prognosis $(49,50)$. It was demonstrated that PADI2 could enhance the effect of nitazoxanide in promoting $\beta$-catenin citrullination and inhibiting Wnt signaling in cancer, and this effect was observed in CRC (51). It was previously identified that FCGBP was associated with immunity as a component of intestinal mucus, which forms the first-line of defense in the gastrointestinal tract $(52,53)$. Previous studies have revealed that transforming growth factor- $\beta$ (TGF- $\beta$ ) molecules were involved in the inhibition of FCGBP expression, which in turn affected the occurrence of various cancer types, including CRC (54), gallbladder cancer (55) and head and neck squamous-cell carcinoma (56). Additionally, cross talk between the Wnt and TGF- $\beta$ signaling pathways has been reported to be involved in regulating epithelial-mesenchymal transition $(57,58)$. Wnt and TGF- $\beta$ were associated with consensus molecular subtypes of CRC (59). Of note, PADI2 and FGCBP were particularly prominent in the present results. In addition, PSAT1 was upregulated in CRC tissue, and overexpression of PSAT1 could promote the progression of CRC $(60,61)$. Based on the HPA database, PADI2 was predicted as an intracellular protein; FCGBP was predicted as a secreted protein and an intracellular protein, while PSAT1 was determined to be a plasma protein and a predicted intracellular protein. Whether these potential targets in the serum were similar to those in cancer tissue remain unknown; however, PADI2 and FCGBP may 

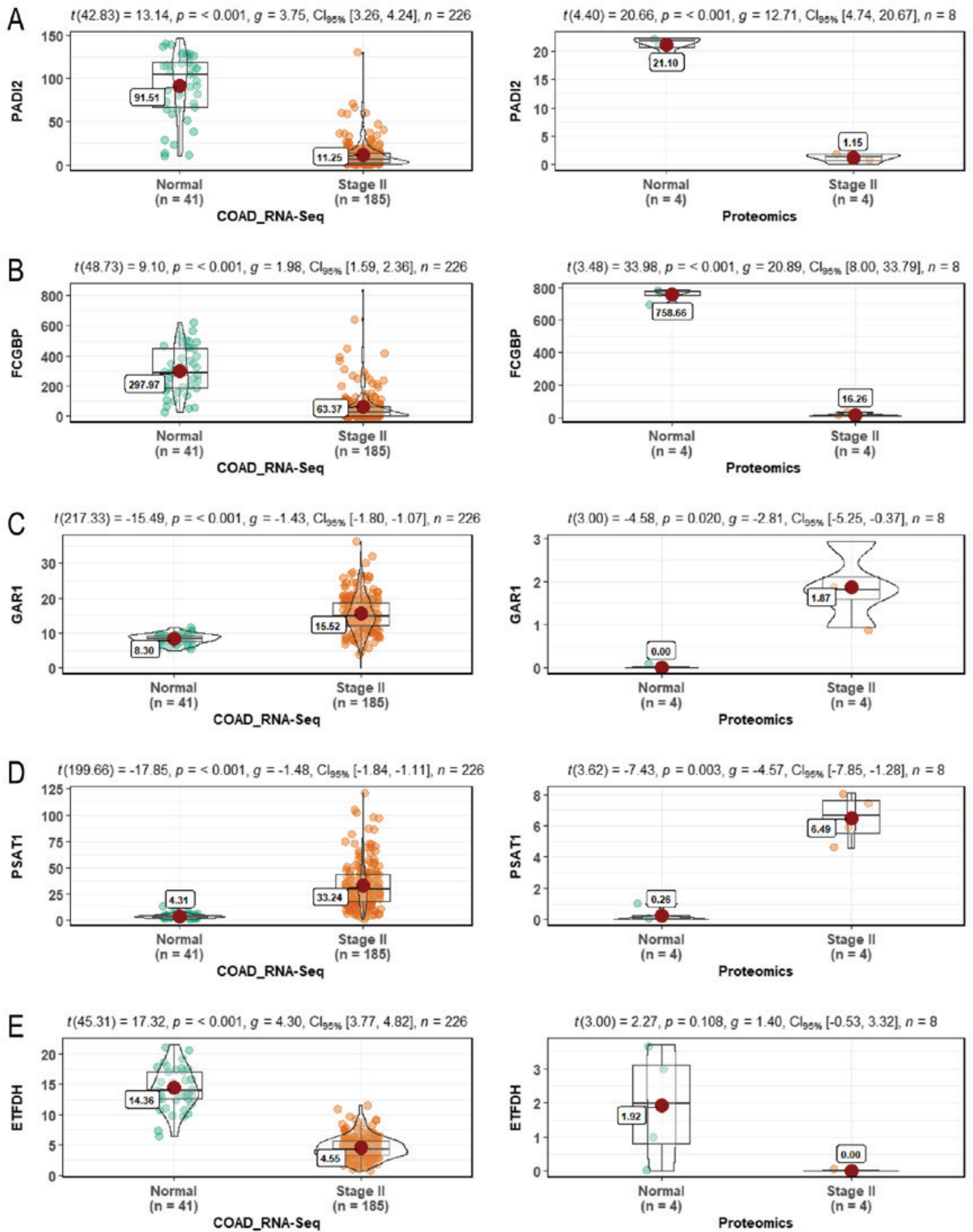

Figure 7. Statistical analysis of critical targets. Statistical analysis of (A) PADI2 ( $\mathrm{P}<0.001$ in RNA-Seq and $\mathrm{P}<0.001$ in proteomics), (B) FCGBP (P<0.001 in RNA-Seq and $\mathrm{P}<0.001$ in proteomics), (C) GAR1 ( $\mathrm{P}<0.001$ in RNA-Seq and $\mathrm{P}=0.020$ in proteomics), (D) PSAT1 ( $\mathrm{P}<0.001$ in RNA-Seq and $\mathrm{P}=0.003$ in proteomics) and (E) ETFDH ( $\mathrm{P}<0.001$ in RNA-Seq and $\mathrm{P}=0.108$ in proteomics). PADI2, protein-arginine deaminase type-2; FCGBP, Fc fragment of IgG binding protein; GAR1, GAR1 ribonucleoprotein; PSAT1, phosphoserine aminotransferase; ETFDH, electron-transfer flavoprotein-ubiquinone oxidoreductase; RNA-Seq, RNA-Sequencing; COAD, colon adenocarcinoma.

be considered as potential biomarkers for the prognosis of $\mathrm{CRC}$. There were some limitations of the present study. The proteomics data were derived from Chinese patients and the mRNA expression data were derived from TCGA-COAD (including African American, Caucasian and Asian). While the ethnic differences could have potential effects. Therefore, the analysis was selected to assess the two types of data to ensure that the potential target screened are meaningful. However, functional enrichment analysis of key genes only provides more general results. The function of the gene is instructive for further research. Although experimental validation was not performed in the present study, a single-gene GSEA was conducted to obtain more accurate functional annotations. 

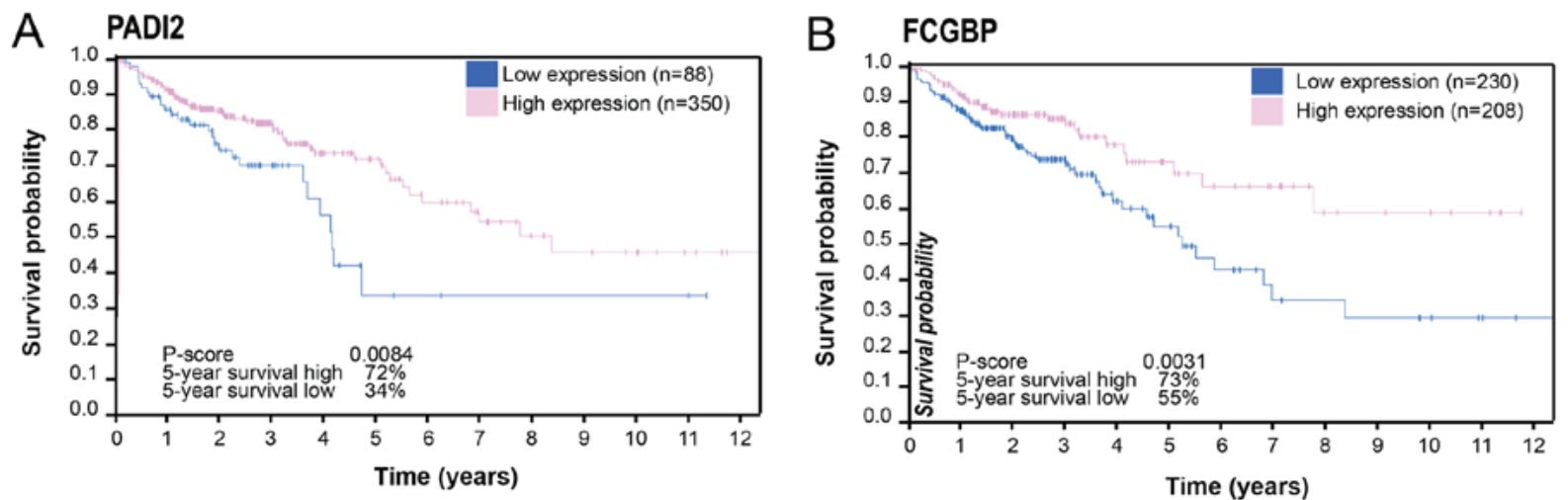

Figure 8. Survival analysis. (A) PADI2 ( $\mathrm{P}=0.0084$ ) was associated with prognosis in stage II CRC. (B) FCGBP ( $\mathrm{P}=0.0031)$ was associated with prognosis in stage II CRC. The information was obtained from the Human Protein Atlas. CRC, colorectal cancer; PADI2, protein-arginine deaminase type-2; FCGBP, Fc fragment of IgG binding protein.
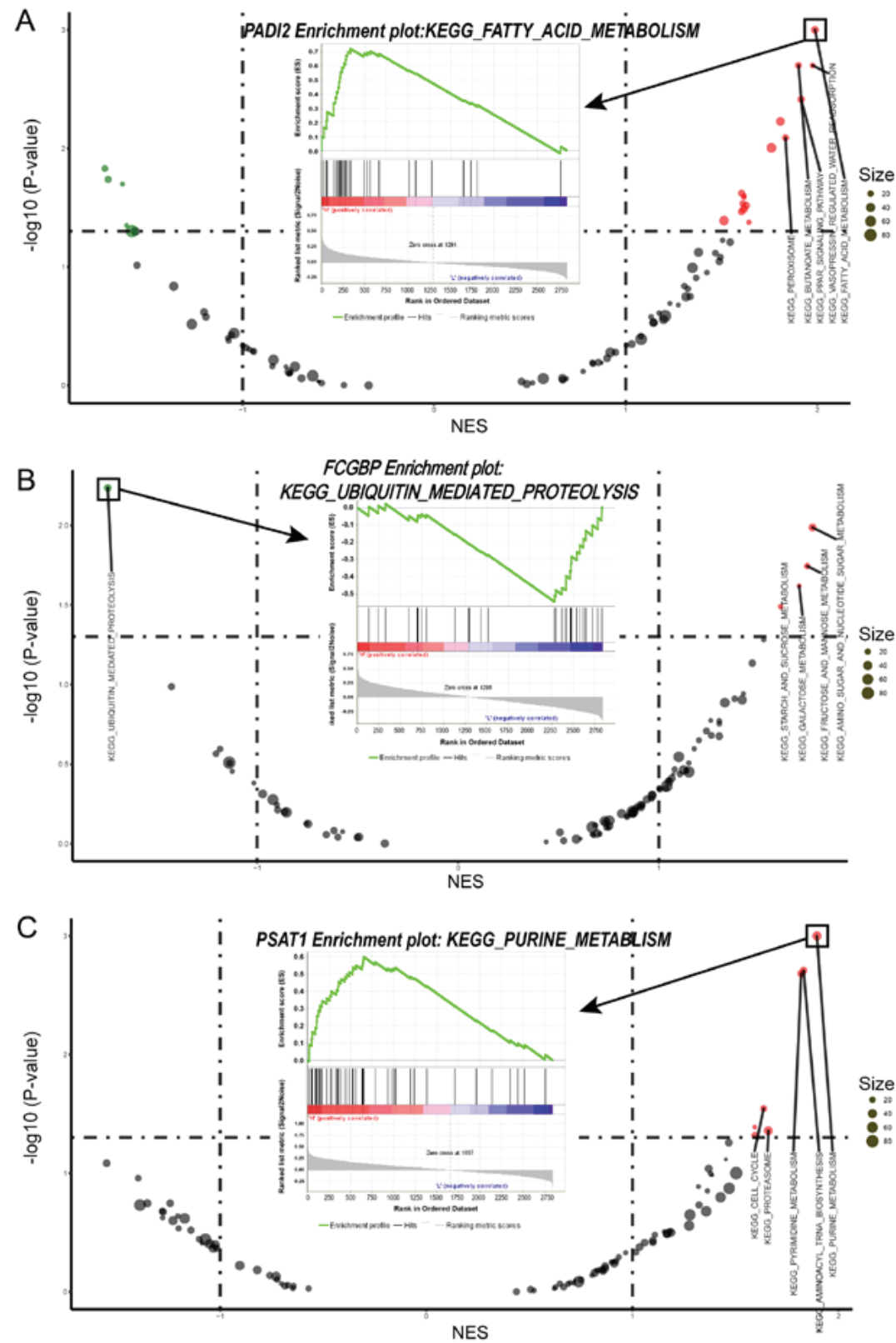

Figure 9. Gene Set Enrichment Analysis of potential targets. (A) Biological function of PADI2. (B) Biological function of FCGBP. (C) Biological function of PSAT1. The size of the dot indicates the number of enriched genes. The red dots indicate that the function was facilitated by the potential targets, while dark green dots indicate that the function was inhibited by potential targets. PADI2, protein-arginine deaminase type-2; FCGBP, Fc fragment of IgG binding protein; PSAT1, phosphoserine aminotransferase; KEGG, Kyoto Encyclopedia of Genes and Genomes; NES, normalized enrichment score. 
In summary, three potential early stage CRC-related targets (PADI2, FCGBP and PSAT1) were identified by combining proteomics and transcriptomics data. These targets could be applied in screening for early stage CRC. Furthermore, the present findings may provide a basis for further investigation into the mechanism underlying the occurrence of CRC.

\section{Acknowledgements}

Not applicable.

\section{Funding}

The present study was funded by the National Natural Science Foundation of China (grant no. 81472030), Jilin Province Science and Technology Department (grant no. 20180101267JC) and Graduate Innovation Fund of Jilin University (grant no. 101832018C070).

\section{Availability of data and materials}

The mRNA expression profiles of colon adenocarcinoma were downloaded from The Cancer Genome Atlas (https:// cancergenome.nih.gov/).

\section{Authors' contributions}

NL, KZ and TL conceived and designed the experiments. WY, JS, YZ and FZ performed the experiments. WY analyzed the data. WY and JS wrote the manuscript. WY, JS, TL, FZ, KZ and NL modified the manuscript.

\section{Ethics approval and consent to participate}

Patients provided informed consent before surgery. The present study was approved by The Clinical Research Ethics Committee of the Second Hospital of Jilin University.

\section{Patient consent for publication}

All patients have provided written informed consent for the publication of any associated data and accompanying images.

\section{Competing interests}

The authors declare that they have no competing interests.

\section{References}

1. Brody H: Colorectal cancer. Nature 521: S1, 2015.

2. Ferlay J, Soerjomataram I, Dikshit R, Eser S, Mathers C, Rebelo M, Parkin DM, Forman D and Bray F: Cancer incidence and mortality worldwide: Sources, methods and major patterns in GLOBOCAN 2012. Int J Cancer 136: E359-E386, 2015.

3. Arnold M, Sierra MS, Laversanne M, Soerjomataram I, Jemal A and Bray F: Global patterns and trends in colorectal cancer incidence and mortality. Gut 66: 683-691, 2017.

4. Weinberg BA, Marshall JL and Salem ME: The Growing Challenge of Young Adults With Colorectal Cancer. Oncology (Williston Park) 31: 381-389, 2017.

5. Bray F, Ferlay J, Soerjomataram I, Siegel RL, Torre LA and Jemal A: Global cancer statistics 2018: GLOBOCAN estimates of incidence and mortality worldwide for 36 cancers in 185 countries. CA Cancer J Clin 68: 394-424, 2018.
6. Osterman E and Glimelius B: Recurrence risk after up-to-date colon cancer staging, surgery, and pathology: Analysis of the entire Swedish population. Dis Colon Rectum 61: 1016-1025, 2018

7. Roncucci L and Mariani F: Prevention of colorectal cancer: How many tools do we have in our basket? Eur J Intern Med 26: 752-756, 2015.

8. Aran V, Victorino AP, Thuler LC and Ferreira CG: Colorectal cancer: Epidemiology, disease mechanisms and interventions to reduce onset and mortality. Clin Colorectal Cancer 15: 195-203, 2016.

9. Stoffel EM and Boland CR: Genetics and genetic testing in hereditary colorectal cancer. Gastroenterology 149: 1191-1203.e2, 2015.

10. Jones PA and Baylin SB: The epigenomics of cancer. Cell 128: 683-692, 2007.

11. Naldini L: Gene therapy returns to centre stage. Nature 526: 351-360, 2015.

12. Wu L, Johnson M and Sato M: Transcriptionally targeted gene therapy to detect and treat cancer. Trends Mol Med 9: 421-429, 2003.

13. Mair B, Kubicek S and Nijman SM: Exploiting epigenetic vulnerabilities for cancer therapeutics. Trends Pharmacol Sci 35: 136-145, 2014.

14. Dalerba P, Sahoo D, Paik S, Guo X, Yothers G, Song N, Wilcox-Fogel N, Forgó E, Rajendran PS, Miranda SP, et al: $\mathrm{CDX} 2$ as a prognostic biomarker in stage II and stage III colon cancer. N Engl J Med 374: 211-222, 2016.

15. Mo A, Jackson S, Varma K, Carpino A, Giardina C, Devers TJ and Rosenberg DW: Distinct transcriptional changes and epithelial-stromal interactions are altered in early-stage colon cancer development. Mol Cancer Res 14: 795-804, 2016.

16. Lucena-Cacace A, Otero-Albiol D, Jiménez-García MP, Muñoz-Galvan S and Carnero A: NAMPT is a potent oncogene in colon cancer progression that modulates cancer stem cell properties and resistance to therapy through Sirt1 and PARP. Clin Cancer Res 24: 1202-1215, 2018.

17. Tomczak K, Czerwińska P and Wiznerowicz M: The Cancer Genome Atlas (TCGA): An immeasurable source of knowledge. Contemp Oncol (Pozn) 19: A68-A77, 2015.

18. Uhlén M, Fagerberg L, Hallström BM, Lindskog C, Oksvold P, Mardinoglu A, Sivertsson Å, Kampf C, Sjöstedt E, Asplund A, et al: Proteomics. Tissue-based map of the human proteome. Science 347: 1260419, 2015.

19. Thul PJ, Åkesson L, Wiking M, Mahdessian D, Geladaki A, Ait Blal H, Alm T, Asplund A, Björk L, Breckels LM, et al: A subcellular map of the human proteome. Science 356: 356, 2017.

20. Uhlen M, Zhang C, Lee S, Sjöstedt E, Fagerberg L, Bidkhori G, Benfeitas R, Arif M, Liu Z, Edfors F, et al: A pathology atlas of the human cancer transcriptome. Science 357: 357, 2017.

21. Langfelder P and Horvath S: WGCNA: An R package for weighted correlation network analysis. BMC Bioinformatics 9: 559, 2008.

22. Sun N, Sun W, Li S, Yang J, Yang L, Quan G, Gao X, Wang Z, Cheng X, Li Z, et al: Proteomics analysis of cellular proteins co-immunoprecipitated with nucleoprotein of influenza A virus (H7N9). Int J Mol Sci 16: 25982-25998, 2015

23. Davis CA, Hitz BC, Sloan CA, Chan ET, Davidson JM, Gabdank I, Hilton JA, Jain K, Baymuradov UK, Narayanan AK, et al: The Encyclopedia of DNA elements (ENCODE): Data portal update. Nucleic Acids Res 46: D794-D801, 2018.

24. Colaprico A, Silva TC, Olsen C, Garofano L, Cava C, Garolini D, Sabedot TS, Malta TM, Pagnotta SM, Castiglioni I, et al: TCGAbiolinks: An R/Bioconductor package for integrative analysis of TCGA data. Nucleic Acids Res 44: e71, 2016.

25. Robinson MD, McCarthy DJ and Smyth GK: edgeR: A Bioconductor package for differential expression analysis of digital gene expression data. Bioinformatics 26: 139-140, 2010.

26. McCarthy DJ, Chen Y and Smyth GK: Differential expression analysis of multifactor RNA-Seq experiments with respect to biological variation. Nucleic Acids Res 40: 4288-4297, 2012.

27. Ito K and Murphy D: Application of ggplot2 to pharmacometric graphics. CPT Pharmacometrics Syst Pharmacol 2: e79, 2013.

28. Gene Ontology Consortium: Gene Ontology Consortium: Going forward. Nucleic Acids Res 43: D1049-D1056, 2015.

29. Yu G, Wang LG, Han Y and He QY: clusterProfiler: An R package for comparing biological themes among gene clusters. OMICS 16: 284-287, 2012.

30. Szklarczyk D, Morris JH, Cook H, Kuhn M, Wyder S, Simonovic M, Santos A, Doncheva NT, Roth A, Bork P, et al: The STRING database in 2017: Quality-controlled protein-protein association networks, made broadly accessible. Nucleic Acids Res 45: D362-D368, 2017. 
31. von Mering C, Huynen M, Jaeggi D, Schmidt S, Bork P and Snel B: STRING: A database of predicted functional associations between proteins. Nucleic Acids Res 31: 258-261, 2003.

32. Kanehisa M and Goto S: KEGG: Kyoto encyclopedia of genes and genomes. Nucleic Acids Res 28: 27-30, 2000.

33. Mootha VK, Lindgren CM, Eriksson KF, Subramanian A, Sihag S, Lehar J, Puigserver P, Carlsson E, Ridderstråle M, Laurila E, et al: PGC-1alpha-responsive genes involved in oxidative phosphorylation are coordinately downregulated in human diabetes. Nat Genet 34: 267-273, 2003.

34. Subramanian A, Tamayo P, Mootha VK, Mukherjee S, Ebert BL, Gillette MA, Paulovich A, Pomeroy SL, Golub TR LanderES, etal: Gene setenrichment analysis: A knowledge-based approach for interpreting genome-wide expression profiles. Proc Natl Acad Sci USA 102: 15545-15550, 2005.

35. Xu H, Wang $\mathrm{C}$, Song $\mathrm{H}, \mathrm{Xu} \mathrm{Y}$ and Ji G: RNA-Seq profiling of circular RNAs in human colorectal Cancer liver metastasis and the potential biomarkers. Mol Cancer 18: 8, 2019.

36. Li XN, Wang ZJ, Ye CX, Zhao BC, Li ZL and Yang Y: RNA sequencing reveals the expression profiles of circRNA and indicates that circDDX17 acts as a tumor suppressor in colorectal cancer. J Exp Clin Cancer Res 37: 325, 2018.

37. Jones MF, Hara T, Francis P, Li XL, Bilke S, Zhu Y, Pineda M, Subramanian M, Bodmer WF and Lal A: The CDX1-microRNA-215 axis regulates colorectal cancer stem cell differentiation. Proc Natl Acad Sci USA 112: E1550-E1558, 2015.

38. Triner D, Devenport SN, Ramakrishnan SK, Ma X, Frieler RA, Greenson JK, Inohara N, Nunez G, Colacino JA, Mortensen RM, et al: Neutrophils restrict tumor-associated microbiota to reduce growth and invasion of colon tumors in mice. Gastroenterology 156: 1467-1482, 2019.

39. Wu L, Saxena S, Awaji M and Singh RK: Tumor-associated neutrophils in cancer: Going Pro. Cancers (Basel) 11: 11, 2019.

40. Galdiero MR, Bianchi P, Grizzi F, Di Caro G, Basso G, Ponzetta A Bonavita E, Barbagallo M, Tartari S, Polentarutti N, et al: Occurrence and significance of tumor-associated neutrophils in patients with colorectal cancer. Int J Cancer 139: 446-456, 2016.

41. Ning C, Li YY, Wang Y, Han GC, Wang RX, Xiao H, Li XY, Hou CM, Ma YF, Sheng DS, et al: Complement activation promotes colitis-associated carcinogenesis through activating intestinal IL-1ß/IL-17A axis. Mucosal Immunol 8: 1275-1284, 2015.

42. Piao C, Zhang WM, Li TT, Zhang CC, Qiu S, Liu Y, Liu S, Jin M, Jia LX, Song WC, et al: Complement 5a stimulates macrophage polarization and contributes to tumor metastases of colon cancer. Exp Cell Res 366: 127-138, 2018.

43. Piao C, Cai L, Qiu S, Jia L, Song W and Du J: Complement 5a enhances hepatic metastases of colon cancer via monocyte chemoattractant protein-1-mediated inflammatory cell infiltration. J Biol Chem 290: 10667-10676, 2015.

44. Wikberg ML, Ling A, Li X, Öberg A, Edin S and Palmqvist R: Neutrophil infiltration is a favorable prognostic factor in early stages of colon cancer. Hum Pathol 68: 193-202, 2017.

45. Governa V, Trella E, Mele V, Tornillo L, Amicarella F, Cremonesi E, Muraro MG, Xu H, Droeser R, Däster SR, et al: The interplay between neutrophils and $\mathrm{CD}^{+} \mathrm{T}$ cells improves survival in human colorectal cancer. Clin Cancer Res 23: 3847-3858, 2017.

46. Zhou G, Peng K, Song Y, Yang W, Shu W, Yu T, Yu L, Lin M, Wei Q, Chen C, et al: CD177+ neutrophils suppress epithelial cell tumourigenesis in colitis-associated cancer and predict good prognosis in colorectal cancer. Carcinogenesis 39: 272-282, 2018

47. Guo W, Zheng Y, Xu B, Ma F, Li C, Zhang X, Wang Y and Chang $\mathrm{X}$ : Investigating the expression, effect and tumorigenic pathway of PADI2 in tumors. OncoTargets Ther 10: 1475-1485, 2017.
48. Funayama R, Taniguchi H, Mizuma M,Fujishima F, Kobayashi M, Ohnuma S, Unno $M$ and Nakayama K: Protein-arginine deiminase 2 suppresses proliferation of colon cancer cells through protein citrullination. Cancer Sci 108: 713-718, 2017.

49. Cantariño N, Musulén E, Valero V, Peinado MA, Perucho M, Moreno V, Forcales SV, Douet J and Buschbeck M: Downregulation of the deiminase PADI2 is an early event in colorectal carcinogenesis and indicates poor prognosis. Mol Cancer Res 14: 841-848, 2016.

50. Shangkuan WC, Lin HC, Chang YT, Jian CE, Fan HC, Chen KH, Liu YF, Hsu HM, Chou HL, Yao CT, et al: Risk analysis of colorectal cancer incidence by gene expression analysis. PeerJ 5: e3003, 2017.

51. Qu Y, Olsen JR, Yuan X, Cheng PF, Levesque MP, Brokstad KA, Hoffman PS, Oyan AM, Zhang W, Kalland KH, et al: Small molecule promotes $\beta$-catenin citrullination and inhibits Wnt signaling in cancer. Nat Chem Biol 14: 94-101, 2018.

52. Pelaseyed T, Bergström JH, Gustafsson JK, Ermund A, Birchenough GM, Schütte A, van der Post S, Svensson F, Rodríguez-Piñeiro AM, Nyström EE, et al: The mucus and mucins of the goblet cells and enterocytes provide the first defense line of the gastrointestinal tract and interact with the immune system. Immunol Rev 260: 8-20, 2014.

53. Johansson ME, Thomsson KA and Hansson GC: Proteomic analyses of the two mucus layers of the colon barrier reveal that their main component, the Muc2 mucin, is strongly bound to the Fcgbp protein. J Proteome Res 8: 3549-3557, 2009.

54. Lee S, Bang S, Song K and Lee I: Differential expression in normal-adenoma-carcinoma sequence suggests complex molecular carcinogenesis in colon. Oncol Rep 16: 747-754, 2006.

55. Xiong L, Wen Y, Miao X and Yang Z: NT5E and FcGBP as key regulators of TGF-1-induced epithelial-mesenchymal transition (EMT) are associated with tumor progression and survival of patients with gallbladder cancer. Cell Tissue Res 355: 365-374, 2014.

56. Wang Y, Liu Y, Liu H, Zhang Q, Song H, Tang J, Fu J and Wang X: FcGBP was upregulated by HPV infection and correlated to longer survival time of HNSCC patients. Oncotarget 8: 86503-86514, 2017

57. Zoni E, van der Pluijm G, Gray PC and Kruithof-de Julio M: Epithelial plasticity in cancer: Unmasking a MicroRNA Network for TGF- $\beta-$-, Notch-, and Wnt-Mediated EMT. J Oncol 2015: 198967, 2015.

58. Chiu HC, Li CJ, Yiang GT, Tsai AP and Wu MY: Epithelial to mesenchymal transition and cell biology of molecular regulation in endometrial carcinogenesis. J Clin Med 8: 8, 2019.

59. Guinney J, Dienstmann R, Wang X, de Reyniès A, Schlicker A, Soneson C, Marisa L, Roepman P, Nyamundanda G, Angelino P, et al: The consensus molecular subtypes of colorectal cancer. Nat Med 21: 1350-1356, 2015.

60. Vié N, Copois V, Bascoul-Mollevi C, Denis V, Bec N, Robert B, Fraslon C, Conseiller E, Molina F, Larroque C, et al: Overexpression of phosphoserine aminotransferase PSAT1 stimulates cell growth and increases chemoresistance of colon cancer cells. Mol Cancer 7: 14, 2008.

61. Ma L, Tao Y, Duran A, Llado V, Galvez A, Barger JF, Castilla EA, Chen J, Yajima T, Porollo A, et al: Control of nutrient stress-induced metabolic reprogramming by $\mathrm{PKC} \zeta$ in tumorigenesis. Cell 152: 599-611, 2013.

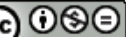

This work is licensed under a Creative Commons Attribution-NonCommercial-NoDerivatives 4.0 International (CC BY-NC-ND 4.0) License. 